\title{
Molde de recorte da placa periestomal: possibilidades de aplicação de novos produtos
}

\section{Peristomal plate cut mold: possibilities for applying new products \\ Molde de recorte de la placa periestomal: posibilidades de aplicación de nuevos productos}

Claudini Lacerda Marques de Freitas Sichieri', Diba Maria Sebba Tosta Souza², Ana Beatriz Alkmim Teixeira-Loyola², Manoel Araújo Teixeira ${ }^{2}$

ORCID IDS

Sichieri CLMF (iD https://orcid.org/0000-0001-5138-4743 Souza DMST (iD https://orcid.org/0000-0002-4743-2455 Teixeira-Loyola ABA (iD https://orcid.org/0000-0002-9089-7012 Teixeira MA (D) https://orcid.org/0000-0002-8088-2351

\section{COMO CITAR}

Sichieri CLMF, Souza DMST, Teixeira-Loyola ABA, Teixeira MA. Molde de recorte da placa periestomal: possibilidades de aplicação de novos produtos. ESTIMA, Braz. J. Enterostomal Ther., 17: e1019. https://doi. org/10.30886/estima.v17.678_PT

\begin{abstract}
RESUMO
Introdução: Dermatite periestomal pode ser causada por vazamentos entre placa adesiva e pele. Objetivo: Descrever o molde de recorte da placa periestomal (MRPP) para avaliação simultânea de novos produtos para tratamento de dermatite. Métodos: Relato de experiência sobre a criação de modelo de recorte, com tamanho e largura suficientes para exposição de parte da pele com dermatite, para aplicação de outro produto. O recorte, em forma trapezoidal e, em acordo com as posições 3, 6, 9 ou 12 horas, correspondendo a um dos quadrantes, foi realizado na placa de carboximetilcelulose, pectina e gelatina acoplada à bolsa para estomas. A parte íntegra da placa aderida à pele adequou-se ao produto-controle. Na análise fotográfica, utilizou-se CorelDRAW 19.0. As lesões foram mensuradas em pixels. Resultado: O recorte não interferiu na aderência da placa do dispositivo de duas peças, possibilitou a aplicação e teste simultâneo de novo produto e a comparação da cicatrização com a placa. Conclusão: O MRPP é uma nova estratégia para estudos da aplicação de outros produtos para dermatite periestomal de forma simultânea no mesmo paciente e demonstra resultados positivos em seu emprego.
\end{abstract}

DESCRITORES: Cicatrização; Dermatite; Estoma Cirúrgico; Processamento de Imagem Assistida por Computador; Tecnologia; Fotografia; Estomaterapia.

\footnotetext{
1.Prefeitura Municipal de Divinópolis - Secretaria Municipal de Saúde - Diretoria de Urgência e Emergência - Divinópolis/MG - Brasil. 2.Universidade do Vale do Sapucaí - Mestrado Profissional em Ciências Aplicadas à Saúde - Pouso Alegre/MG - Brasil.

*Autor correspondente: souzadiba@gmail.com

Recebido: 14 Dez 2018 | Aceito: 02 Maio 2019
} 


\begin{abstract}
Introduction: peristomal dermatitis can be caused by leakage between adhesive plate and skin. Objective: to describe the peristomal plate cut mold (PPCM) for simultaneous evaluation of new products for the treatment of dermatitis. Methods: experience report about creation of a cut model, with sufficient size and width to expose part of the skin with dermatitis, to apply another product. The trapezoidal cut, according to positions 3, 6, 9 or 12 hours, corresponding to one of the quadrants, it was performed on the plate of carboxymethylcellulose, pectin, and gelatine coupled to the stoma bag. The entire part of the plate adhered to the skin was adapted to the control product. In the photographic analysis, CoreIDRAW 19.0 was used. The lesions were measured in pixels. Result: the cut did not interfere with the adhesion of two parts device board, it made possible the simultaneous application and testing of a new product and the comparison of the healing with the plate. Conclusion: the PPCM is a new strategy for studies of the application of other products for peristomal dermatitis simultaneously in the same patient and demonstrates positive results in its use.
\end{abstract}

DESCRIPTORS: Healing; Dermatitis; Surgical Stoma; Computer Assisted Image Processing; Technology; Photography; Stomatherapy.

\title{
RESUMEN
}

Introducción: Dermatitis perormomal puede ser causada por fugas entre placa adhesiva y piel. Objetivo: Describir el molde de recorte de la placa periestomal (MRPP) para la evaluación simultánea de nuevos productos para el tratamiento de dermatitis. Métodos: Relato de experiencia sobre la creación de modelo de recorte, con tamaño y anchura suficientes para la exposición de parte de la piel con dermatitis, para aplicación de otro producto. El recorte, en forma trapezoidal y, de acuerdo con las posiciones 3, 6, 9 o 12 horas, correspondiendo a uno de los cuadrantes, fue realizado en la placa de carboximetilcelulosa, pectina y gelatina acoplada a la bolsa para estomas. La parte íntegra de la placa adherida a la piel se adecuó al producto-control. En el análisis fotográfico, se utilizó CorelDRAW 19.0. Las lesiones se midieron en píxeles. Resultado: El recorte no interfirió en la adherencia de la placa del dispositivo de dos piezas, posibilitó la aplicación y prueba simultánea de nuevo producto y la comparación de la cicatrización con la placa. Conclusión: El MRPP es una nueva estrategia para estudios de aplicación de otros productos para dermatitis periestomal de forma simultánea en el mismo paciente y demuestra resultados positivos en su empleo.

DESCRIPTORES: Cicatrización; Dermatitis; Estoma Quirúrgicos; Procesamiento de imágenes; Asistida por ordenador; Tecnología; Fotografía.

\section{INTRODUÇÃO}

Dentre as principais causas relacionadas à confecção de estomias à população adulta e idosa no Brasil encontram-se as neoplasias, principalmente o câncer colorretal, cujas estimativas para 2018 o apresentaram como o terceiro mais incidente para os homens, com 17.380 casos novos, e o segundo para as mulheres, com $18.980^{1}$.

Além dos cânceres colorretal e de bexiga, na maioria das vezes, as estomias estão vinculadas às doenças crônicas intestinais e urinárias, às doenças inflamatórias, como a doença de Crohn, a retocolite ulcerativa e a diverticulite, e ao tratamento de enfermidades congênitas e trauma abdominal ${ }^{2}$

A confecção de uma estomia é considerada procedimento comum e é realizada por diversas especialidades cirúrgicas. Ainda que comumente processada, apresenta complicações subestimadas em sua maioria ${ }^{3}$. Existem avanços nas técnicas cirúrgicas e aprimoramento de práticas e de produtos, contudo, $70 \%$ das pessoas com estomia apresentaram complicações, sendo a dermatite periestomal a de maior prevalência e suas causas são variadas ${ }^{4}$.
O fator mais comum dessa doença é a inadequada adesão do equipamento coletor à parede abdominal, ocasionado o vazamento de efluente sobre a pele periestomal. As razões que contribuem para seu desenvolvimento estão associadas, em sua maioria, à má localização da estomia ou, ainda, à escolha equivocada do sistema coletor ${ }^{6}$.

Assim, medidas preventivas e de tratamento fazem-se necessárias, dentre elas o afastamento da causa raiz, o incentivo ao autocuidado da pele e a aplicação correta e indicada de coberturas para o reestabelecimento da pele periestomal ${ }^{7}$. A perda de controle das eliminações em pessoas com estomia as obriga ao uso de dispositivos coletores, que são vários, porém todos têm uma placa adesiva protetora da pele e uma bolsa para armazenamento do efluente. Alguns dispositivos são compostos por duas peças (placa e bolsa) e outros de peça única na qual a bolsa é inseparável da placa ${ }^{8}$.

Apesar da tecnologia aplicada na confecção desses dispositivos, por se tratar de produtos aderentes à pele, podem ocorrer vazamentos entre a placa adesiva e a 
pele, assim como entre a placa e a bolsa (no caso de dispositivo de duas peças), o que, por períodos prolongados, devido à característica do efluente, podem lesar a pele periestomal ${ }^{8}$.

Considera-se a cobertura todo o material, substância ou produto que se aplica sobre uma ferida, formando uma barreira física com capacidade de proteção e regeneração celular. O tratamento eficaz de qualquer lesão subentende, dentre outras precauções, a escolha do tipo de cobertura mais apropriada para cada tipo específico de ferida, nas diferentes situações clínicas e cirúrgicas ${ }^{9}$. As coberturas podem ser classificadas como primária, aplicada diretamente na lesão, e secundária, aplicada sobre a cobertura primária ${ }^{10}$.

Embora o processo de reparação tecidual seja sistêmico, para que seja efetivo, é necessário favorecer as condições locais da lesão com tratamento tópico apropriado. Nesse sentido, nas últimas décadas, houve uma verdadeira revolução tecnológica baseada em pesquisas científicas que avaliaram biomateriais e produtos diversos para o tratamento de feridas ${ }^{7}$.

Mesmo com o investimento em pesquisa direcionada ao campo da cicatrização e a crença em que existe excesso de produtos no mercado, em sua maioria com tecnologias patenteadas por multinacionais, o aperfeiçoamento de tais recursos é essencial não só para tornar esses recursos mais baratos e acessíveis ao maior número de pessoas possível, mas, também, para possibilitar o aproveitamento de matériasprimas encontradas em todas as regiões do Brasil ${ }^{11}$.

Com o aumento da necessidade de se validar a efetividade terapêutica, mensurações objetivas são primordiais ao cuidado atual da saúde ${ }^{12}$. A rápida mudança no campo das ciências da saúde faz com que a formação inicial dos profissionais fique rapidamente obsoleta. Novos equipamentos e tecnologias, bem como procedimentos e novas práticas, são áreas as quais requerem atualização de conhecimentos ${ }^{13}$.

Nesse contexto, o recorte na placa da bolsa coletora permite a aplicação de novos produtos no paciente com dermatite periestomal, avaliando produtos distintos para o tratamento dessa afecção.

\section{OBJETIVO}

O presente estudo tem como objetivo descrever o molde de recorte da placa periestomal (MRPP) para avaliação simultânea de novos produtos para tratamento da dermatite.

\section{MÉTODOS}

Relato de experiência aprovado pelo comitê de ética (CAAE 69729217.2.0000.5102 sob o parecer no 064826/2017) sobre a criação de um modelo de recorte com tamanho e largura suficientes para exposição de parte da pele com dermatite à aplicação de outro produto. Esse molde de recorte foi realizado em forma trapezoidal e, em acordo com as posições 3, 6, 9 ou 12 horas, correspondendo a um dos quadrantes. Foi realizado na placa de carboximetilcelulose, pectina e gelatina acoplada à bolsa para estomias.

O MRPP foi aplicado em duas pessoas com colostomia que aceitaram e assinaram o Termo de Consentimento Livre e Esclarecido.

O recorte deixa parte da pele periestomal exposta e possibilita a aplicação de um produto e a observação da possível interferência do recorte na aderência da placa e na avaliação das propriedades cicatriciais dos dois produtos. Os procedimentos para realização do molde foram realizados em quatro etapas:

1. Na pele periestomal com presença de dermatite, realizou-se a limpeza com solução fisiológica a 0,9\% em temperatura morna (Fig. 1a).

2. Feito o recorte na placa de carboximetilcelulose, gelatina e pectina, a pele periestomal em que foi aplicado o novo produto ficou exposta. A área do recorte correspondeu à área de estudo a fim de avaliar a cicatrização. A parte íntegra (sem o recorte) correspondeu à área controle (Fig. 1b).

3. A placa com a área de recorte (estudo) e a área da placa (controle) foi fixada à pele (Fig. 1b).

4. Após a fixação na área do recorte, aplicou-se um novo produto (estudo) (Fig. 1c).

O cálculo do percentual foi realizado em quatro etapas:

1. Realizou-se o cálculo da área total da lesão em pixels por meio do programa CorelDRAW.

2. Realizou-se o cálculo das áreas de aplicação do produto-estudo e de aplicação do produto-controle, separadamente, em pixels.

3. Os valores das áreas do grupo-estudo e do grupocontrole foram convertidos em percentuais de áreas.

4. Fez-se o cálculo da diferença percentual das áreas iniciais (registro fotográfico do dia 0) e finais (registro fotográfico do dia 8) de cada local (Fig. 2).

As fotografias foram feitas em ângulo de $90^{\circ}$ a uma distância de $25 \mathrm{~cm}$. Após as mensurações em pixel embasadas por Tacani et al. ${ }^{14}$ para avaliar a cicatrização, as unidades de 
pixels foram convertidas em porcentagem pelo programa Microsoft Excel 2013. Chegou-se ao resultado final percentual de áreas de estudo e controle, separadamente.

Os resultados dos registros fotográficos utilizados na análise foram efetuados nos dias 0 e 8 das áreas de aplicação do produto-estudo e do produto-controle.

O modelo de medida do recorte da bolsa foi avaliado em dois participantes com colostomia em acompanhamento no Centro de Especialidades Médicas de Divinópolis, estado de Minas Gerais, em uso de dispositivo coletor de duas peças e que apresentaram dermatite periestomal com lesão de espessura parcial.

O produto utilizado foi um gel fitoterápico que se encontra em processo de registro no Instituto Nacional de Propriedade Industrial (INPI), o qual foi comparado à própria base da bolsa coletora - produto convencional de carboximetilcelulose, gelatina e pectina - que se adere à pele. Os registros fotográficos analisados foram feitos nos dias 0 e 8 da pesquisa. O recorte foi feito na placa com barreira protetora de pele. Essa barreira protetora é, também, a própria placa de encaixe da bolsa coletora do dispositivo.

\section{RESULTADOS}

$\mathrm{Na}$ Tabela 1 estão descritos os resultados da análise percentual da redução das lesões das áreas de estudo e controle dos participantes 1 e 2 e na Fig. 3 está relatada a evolução da cicatrização de ambas as áreas, estudo e controle do participante 2 .

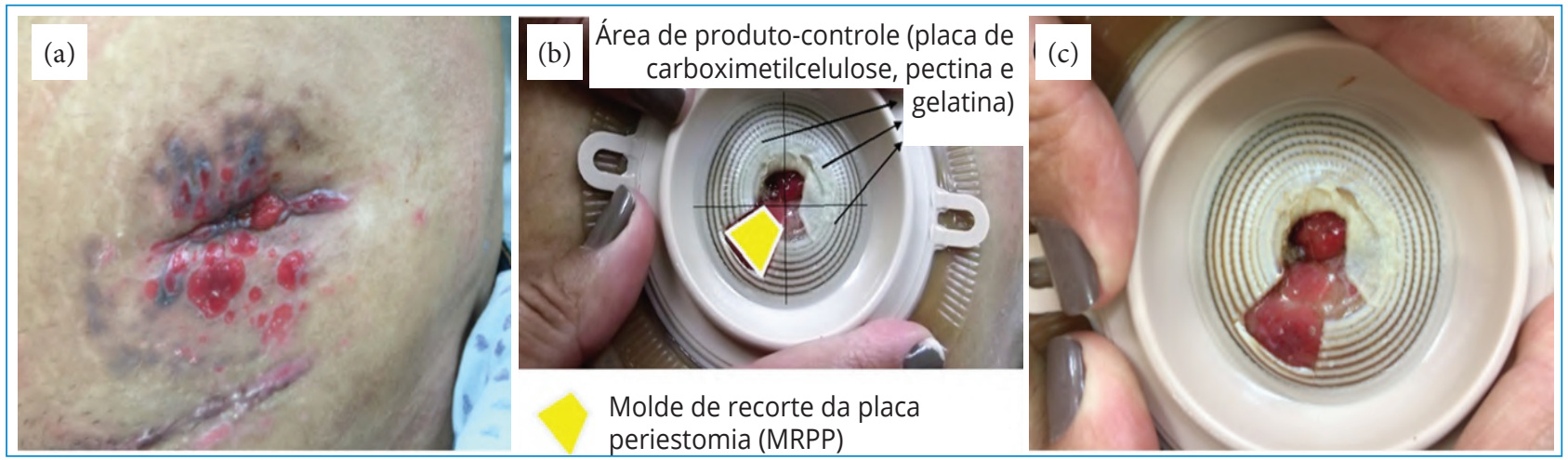

Figura 1. (a) Dermatite periestoma após a limpeza, (b) $2^{\circ}$ e $3^{\circ}$ etapas do MRPP, (c) $4^{a}$ etapa do MRPP demonstrativo do produto aplicado. Divinópolis, estado de Minas Gerais, Brasil.
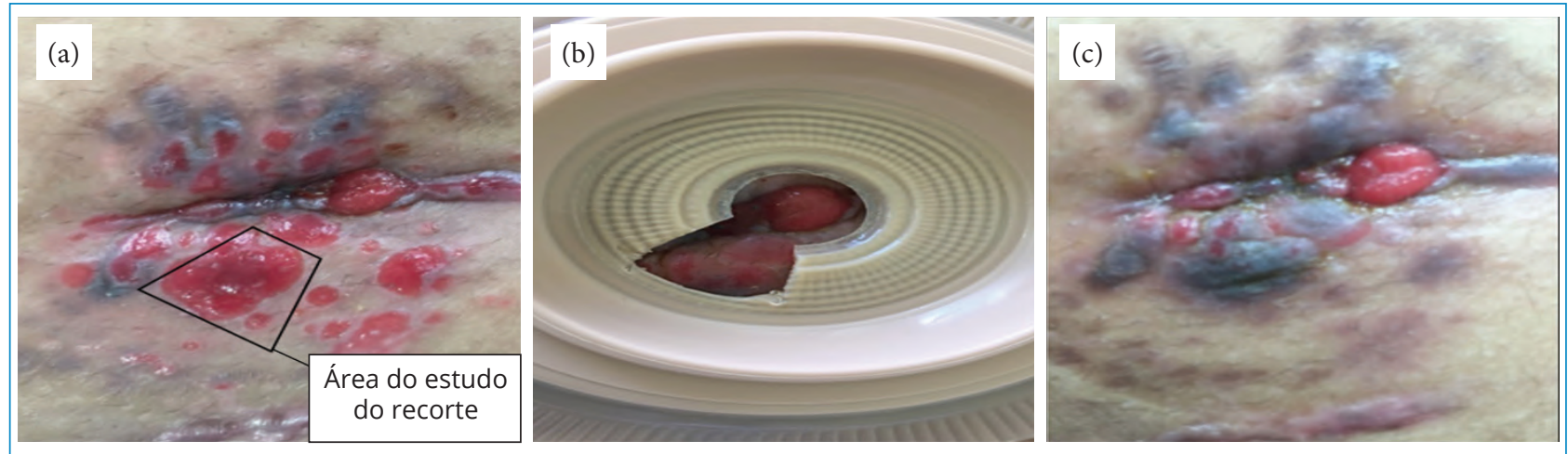

Figura 2. (a) Área do estudo do recorte, (b) Participante 1 no dia 0, (c) Participante 1 no dia 8, Divinópolis, estado de Minas Gerais, Brasil.

Tabela 1. Descrição da análise percentual da redução das lesões das áreas de estudo e controle dos participantes 1 e 2 com colostomia. Divinópolis, estado de Minas Gerais, Brasil.

\begin{tabular}{|c|c|c|c|c|}
\hline \multirow{2}{*}{ Participante } & \multirow{2}{*}{ Descrição } & \multirow{2}{*}{$\begin{array}{c}\text { Dia } 0 \\
\text { Área inicial }\end{array}$} & \multicolumn{2}{|c|}{ Dia 8} \\
\hline & & & Área final & Redução \\
\hline 1 & Área de estudo & 100,0 & 12,1 & 87,8 \\
\hline 1 & Área de controle & 100,0 & 11,4 & 88,5 \\
\hline 2 & Área de estudo & 100,0 & 0,0 & 100,0 \\
\hline 2 & Área de controle & 100,0 & 0,0 & 100,0 \\
\hline
\end{tabular}




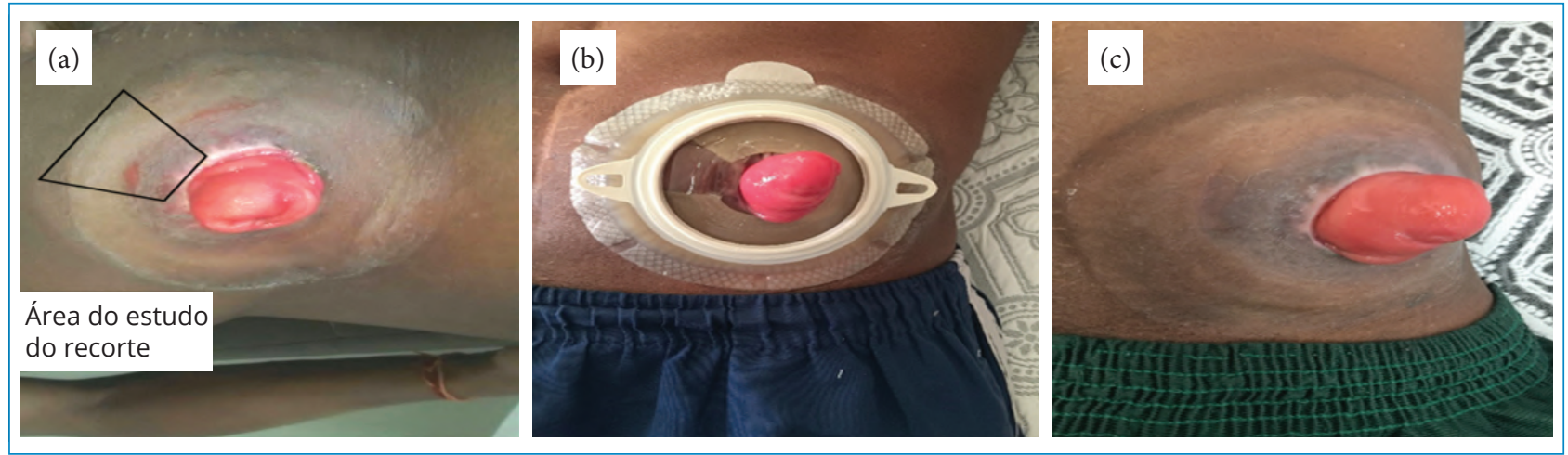

Figura 3. (a) Área do estudo do recorte, (b) Participante 2 no dia 0, (c) Participante 2 no dia 8, Divinópolis, estado de Minas Gerais, Brasil.

\section{DISCUSSÃO}

A avaliação das mudanças no tamanho da ferida, ao longo do tempo, é, muitas vezes, considerada um indicador primário de tratamento bem-sucedido. O elevado grau de precisão e reprodutibilidade em técnicas de medição é altamente desejável em situações clínicas e é extremamente importante em estudos de investigação cuja variação no tamanho da ferida é um desfecho primário ${ }^{15}$. Não há um protocolo-padrão para avaliar o tamanho da ferida e monitorar a cicatrização da dermatite periestomal. No presente estudo, desenvolveu-se um molde com recorte que favorece a avaliação de um local em que não era possível analisar-se o processo de cicatrização dessas lesões, uma vez que o portador de estomia não podia ficar sem a bolsa coletora.

$\mathrm{Na}$ análise fotográfica do molde do recorte da placa da bolsa coletora, a fotografia mostrou-se relevante quando as unidades de pixels foram convertidas em porcentagem pelo programa Microsoft Excel 2013, chegando ao resultado final percentual de áreas de estudo e controle, separadamente. Essa estratégia confirma que a imagem da ferida pode ser obtida por meio de câmeras fotográficas digitais ou manuais e tem como vantagem fornecer amostra clara da aparência da ferida, pois sugere seu tamanho, quando utilizada uma régua ao lado da lesão, e serve como parâmetro para avaliações posteriores. Entretanto, não oferece precisão quanto à profundidade e outros aspectos da lesão ${ }^{16}$.

Os mesmos autores destacam a importância de determinados desvelos, como manter sempre a mesma distância e o mesmo ângulo da lesão ao fotografá-la, colocar etiqueta ao seu lado com data e local, solicitar autorização para fotografar por escrito do cliente ou seu responsável, explicando seu objetivo e garantindo a continuidade do tratamento, mesmo que esse não queira mais ser fotografado. A documentação fotográfica deve ser feita em cores e com intervalos de dias, para que se possam avaliar as alterações ao longo do tempo. Dessa forma, pacientes e familiares têm oportunidade de acompanhar a evolução da cicatrização por meio das fotografias, que lhes servem de incentivo e estímulo ao tratamento. Esses detalhes foram observados durante a captação das imagens do molde do recorte da bolsa coletora e durante a avaliação desse novo modelo, a fim de se pesquisar a dermatite periestoma ${ }^{16}$.

Em revisão bibliográfica, constatou-se que a dermatite periestomal vem sendo estudada de forma incipiente, por ser considerada "simples" e sua classificação ser baseada naquelas estabelecidas por outros segmentos do tratamento de lesões de pele. As classificações de dermatite periestomal são propostas genericamente como leve, moderada e grave (severa ou intensa $)^{17}$. Nesta investigação, para avaliar o modelo de um MRPP, avaliaram-se pessoas com dermatites leve à moderada e em uso de bolsa coletora de duas peças, cuja base do dispositivo apresentou-se resistente ao recorte e à aplicação de um novo produto, comprovando que o molde não alterou o uso convencional da bolsa.

Em recente estudo, as autoras identificaram instrumentos internacionais construídos e desenvolvidos para efetivar a classificação das lesões da pele periestomal, porém poucos com propriedades de medidas testadas e, ainda, em menor quantidade, estudos de adaptação cultural e validação para traduções e aplicação em outros idiomas ${ }^{4}$. Destaca-se a importância da testagem dessas propriedades e da validação, tradução e adaptação transcultural desses novos instrumentos que detectam precocemente os fatores de riscos e impedem que essas afecções periestomais se agravem. Esse fato corrobora o presente estudo em que as 
dermatites foram identificadas no início e se apresentavam de forma superficial.

As causas mais comuns das dermatites foram descritas como irritativas ou desencadeadas por processos inflamatórios da pele periestoma em contato com as eliminações e maximizadas por produtos e traumas, fatores que alteram o $\mathrm{pH}$ da pele, como alergias que podem ser ocasionadas pelo próprio dispositivo utilizado (área de resina da placa, por exemplo), por traumas que envolvem técnicas abrasivas de limpeza, assim como pela remoção inadequada dos dispositivos e por fatores relacionados à técnica cirúrgica, comorbidades, idade, dentre outros ${ }^{18}$.

Neste estudo, os participantes foram acometidos por uma ou mais complicações, especialmente as tardias, como a dermatite que ocorre mais de 15 dias após a cirurgia. Também apresentaram alterações da pele, sendo que um apresentou rubor e calor e ambos apresentaram dor e lesão superficial, sinais e sintomas característicos da dermatite periestomal presente na pele da área de estudo e controle, garantindo uniformidade à pesquisa. Merece salientar que ambas as áreas, estudo e controle, apresentaram cicatrização total, sem interferência entre elas e equiparando um produto convencional estabelecido com o novo produto.

Durante a avaliação do método MRPP, não houve interferência na adesão do grupo-controle. Mesmo em meio hostil, com variações de umidade e acidez, o produto do estudo permaneceu aderido à pele por mais de 40 horas, assim como o produto-controle.
Dessa forma, acredita-se que haja necessidade de estudos clínicos com número maior de participantes para concretizar o uso desse recurso inovador e implementar novos produtos para a cicatrização e aderência da área periestoma.

\section{CONCLUSÃO}

O método MRPP foi criado com possibilidades para a avaliação de dois produtos simultâneos ao tratamento de dermatite periestoma com lesões de espessura parcial. Nas pessoas com colostomia e dermatite periestomal, demonstrou-se que a aderência da placa convencional não se altera, viabiliza a aplicação de outro produto na área de estudo e mantém o produto convencional na área-controle na mesma pessoa. O MRPP é uma nova estratégia para estudos de produtos distintos em momentos simultâneos no mesmo paciente e aponta resultados positivos em sua utilização.

\section{CONTRIBUIÇÃO DOS AUTORES}

Conceitualização, Sichieri CLMF e Souza DMST; Metodologia, Sichieri CLMF; Souza DMST; Teixeira-Loyola ABA e Teixeira MA; Investigação, Sichieri CLMF e Souza DMST; Redação - Primeira versão, Sichieri CLMF; Souza DMST; Teixeira-Loyola ABA e Teixeira MA; Redação Revisão \& Edição, Sichieri CLMF e Souza DMST; Recursos, Sichieri CLMF e Souza DMST; Supervisão, Souza DMST..

\section{REFERÊNCIAS}

1. Instituto Nacional de Câncer. Causas e prevenções. Estatística de câncer [Internet]. Rio de Janeiro: INCA; 2018 [citado 10 Maio 2019]. Disponível em: https://www.inca.gov. br/numeros-de-cancer

2. Silva AC, Silva GNS, Cunha RR. Caracterização de pessoas estomizadas atendidas em consulta de enfermagem do serviço de estomoterapia do município de Belém-PA. ESTIMA, Braz J Enterostomal Ther. 2012;10(1):20-7.

3. Santos CHM dos, Bezerra MM, Bezerra FMM, Paraguassú BR. Perfil do paciente ostomizado e complicações relacionadas ao estoma. Rev Bras Coloproct. 2007;27(1):169. https://doi.org/10.1590/S0101-98802007000100002

4. Nunes MLG, Santos VLCG. Instrumentos de avaliação das complicações da pele periestoma: revisão integrativa. Aquichan. 2018;18(2):477-91. https://doi.org/10.5294/aqui.2018.18.4.9
5. Paula PR, Matos D. Complicações precoces e tardias nas estomias intestinais e pele periestomia. In: Santos VLCG, Cesaretti IUR, editores. Assistência em estomaterapia: cuidando de pessoas com estomias. São Paulo: Atheneu; 2015. p. 311-9.

6. Asociación Mexicana de Cirugía General. Guía de pratica clínica basada en evidencia científica para el marcaje y manejo integral de personas adultas con estomas de eliminación [Internet]. México: AMCG; 2011.

7. Diniz RV, Anselmo MN, Soares RAS, Santos IBC, Oliveira MJGS. Problema de pele em paciente estomizada: relato de caso. R Bras Cai Saúde. 2013;17(4):371-6. https://doi. org/10.4034/RBCS.2013.17.04.08

8. Collet JA, Silva FP, Aimone JFL. Bolsas coletoras utilizadas por estomizados: uma análise tridimensional. Design Tecnol. 2016;11:1-10. https://doi.org/10183/151143 
9. Galvão MTG, Alexandre HO, Dantas PB, Lima ICV de, Lopes EM. Uso da fotografia no processo do cuidar: tendências das ações de enfermagem. Cienc Enferm 2013;19(3):31-9. https://doi.org/10.4067/S0717-95532013000300004

10. Blanes L. Curativos. In: Ferreira LM. Guia de cirurgia plástica/ coordenação das seções. Barueri: Manole; 2007. p. 83-89.

11. Mandelbaum SH, Di Santis EP, Mandelbaum MHS. Cicatrização: conceitos atuais e recursos auxiliares - Parte I. An Bras Dermatol. 2003;78(4):393-410. https://doi. org/10.1590/S0365-05962003000400002

12. Carvalho RM de, Perez MCJ, Miranda Júnior F. Volúmetro com câmaras: apresentação de um protótipo de dimensões reduzidas desenvolvido para a avaliação objetiva do volume de mão. J Vasc Bras. 2010;9(2):76-80. https://doi. org/10.1590/S1677-54492010000200011

13. Moran JM, Lavado-Garcia JM, Pedrera-Zamorano JD. Métodos de enfermagem para a medição da composição corporal. Rev Latino-Am Enfermagem. 2011;19(4):1033-8. https://doi.org/10.1590/S0104-11692011000400024
14. Tacani PM, Toguchi PAPM, Machado AFP, Tacani RE, Freitas JOG. Prevalência e tratamento fisioterapêutico de deiscências da ferida operatória após cirurgias plásticas: análise retrospectiva. R Bras Ci Saúde. 2014;12(39):28-34. https://doi.org/10.13037/rbcs.vol12n39.2110

15. Haghpanah S, Bogie K, Wang X, Banks PG, Ho CH. Reliability of electronic versus manual wound measurement techniques. Arch Phy Me Rehabil. 2006;87(10):1396-1402. https://doi. org/10.1016/j.apmr.2006.06.014

16. Oliveira BGRB, Castro JBA, Andrade NC. Técnicas utilizadas na aferição de feridas e avaliação do processo cicatricial. Saúde Coletiva 2005;2(6):57-62.

17. Bosio G, Pisani F, Lucibello L, Fonti A, Scrocca A, Morandell C, et al. A proposal for classifying peristomal skin disorders: results of a multicenter observational study. Ostomy Wound Manage. 2007;53(9):38-43.

18. Santos VLC de G. Como eu trato as dermatites periestoma. Rev Esc Enferm USP. 1994;28(I):67-71. https://doi. org/10.1590/0080-6234199402800100067 